\title{
Uso de corticoides antenatales en fetos de término o cercanos al término: revisión sistemática y meta-análisis de estudios aleatorizados controlados (1)
}

\author{
Saccone G, Berghella V. Antenatal corticosteroids for maturity of term or near term \\ fetuses: systematic review and meta-analysis of randomized controlled trials. BMJ \\ 2016;355:i5044.
}

Análisis Crítico: Fernando Ferrer Márquez, Caterina Solari, Jorge A. Carvajal, PhD.

Unidad de Medicina Materno-Fetal. División de Obstetricia y Ginecología. Escuela de Medicina. Facultad de Medicina. Pontificia Universidad Católica de Chile.

\section{RESUMEN (1)}

Objetivo: Evaluar la eficacia de administrar corticoides prenatales en gestaciones $\geq 34$ semanas. Diseño: Revisión sistemática con meta-análisis. Fuentes de datos: Se realizaron búsquedas desde el inicio del estudio hasta febrero de 2016 en bases de datos electrónicas. Criterios de elegibilidad para la selección de estudios: Fueron seleccionados estudios clínicos que compararon el uso de corticoides versus placebo en mujeres con un embarazo único de $\geq 34$ semanas. Fueron incluidos estudios que utilizaron corticoides entre las 34+0 y las $36+6$ y trabajos que incluyeron la administración de corticoides antes de una cesárea electiva a término ( $\geq 37$ semanas). Síntesis de los datos: El resultado primario fue evaluar la incidencia del síndrome de dificultad respiratoria grave del recién nacido (SDR). Los resultados fueron informados como medias o riesgos relativos con intervalos de confianza del 95\%. Resultados: Se analizaron seis estudios, con 5.698 embarazos únicos. Tres incluyeron 3.200 mujeres con embarazos entre las $34+0$ y $36+6$ semanas con riesgo de parto prematuro inminente al momento del ingreso hospitalario. Los otros tres estudios incluyeron 2.498 mujeres sometidas a cesárea electiva a las $\geq 37$ semanas. Los recién nacidos de madres que recibieron corticoides prenatales en embarazos de $\geq 34$ semanas, tuvieron un riesgo significativamente menor de SDR (riesgo relativo: 0,74; IC95\% entre 0,61 a 0,91), SDR leve (RR: 0,67; IC95\% entre 0,46 a 0,96), SDR moderado (RR: 0,39; IC95\% entre 0,18 a 0,89$)$, taquipnea transitoria del recién nacido (RR: 0,56; IC95\% entre 0,37 a 0,86), SDR grave (RR: 0,55, IC95\% entre 0,33 a 0,91), uso de surfactante y requerimiento de ventilación mecánica y un tiempo significativamente menor de requerimiento de oxígeno (Diferencia de -2,06 horas, con un IC95\% entre $-2,17$ a $-1,95$ horas), menor concentración máxima de oxígeno inspirado $(-0,66 \%$, IC95\% entre $-0,69 \%$ a $-0,63 \%)$, estadía mas corta en unidad de cuidados intensivos neonatales (-7,64 días; IC95\% entre -7,65 a -7,64), puntuación Apgar más altos en comparación con controles. Los recién nacidos de madres que recibieron betametasona entre las 34+0-36+6 semanas de gestación, tenían una incidencia significativamente menor de taquipnea transitoria del recién nacido ( $R R$ : 0,72; IC95\% entre 0,56 a 0,92), SDR grave (RR: 0,60; IC $95 \%$ entre 0,33 a 0,94$)$ y de utilización de drogas vaso-activas (RR: 0,61; IC 95\% entre 0,38 a 0,99). Los recién nacidos de madres que fueron sometidas a cesárea electiva en embarazos $\geq 37$ semanas y que recibieron corticoides 48 horas antes del parto, tuvieron un riesgo significativamente menor de SDR (RR: 0,40; IC95\% entre 0,27 a 0,59), SDR leve (RR: 0,43; IC95\% entre 0,26 a 0,72), SDR moderado (RR: 0,40; IC 95\% entre 0,18 a 0,88), taquipnea transitoria del recién nacido (RR: 0,38; IC95\% entre 0,25 a 0,57), necesidad de ventilación mecánica (RR: 0,19, IC95\% entre 0,08 a 0,43), tiempo significativamente menor de necesidad de oxígeno terapia (diferencia de -2,06 horas; IC95\% entre -2,17 a -1,95 horas), menor porcentaje de concentración máxima de oxígeno inspirado (RR: 
$-0,66 \%$; IC95\% entre $-0,69 \%$ a $-0,63 \%$ ), menor estadía en cuidados intensivos neonatales (RR: -7,44 días; IC95\% entre -7,44 a -7,43 días) y una mayor puntuación de Apgar tanto al primer como a los cinco minutos. Conclusiones: El uso de corticoides prenatales en embarazos $\geq 34$ semanas, reduce la morbilidad respiratoria neonatal. Un curso único de corticoides puede ser considerado en las mujeres con riesgo de un parto prematuro tardío (Entre las 34+0 - 36+6 semanas de gestación), así como para las mujeres sometidas a cesárea electiva a $\geq 37$ semanas de gestación.

\section{ANÁLISIS DE LA INVESTIGACIÓN}

\section{A. Relevancia clínica de la investigación}

El síndrome de dificultad respiratoria (SDR) es una de las causas más importantes de morbilidad y mortalidad neonatal precoz. En Estados Unidos afecta al $20 \%$ de los recién nacidos de bajo peso (<2.500 gramos) y cerca del $30 \%$ en los neonatos con un peso de nacimiento menor a 2.000 gramos. La insuficiencia respiratoria en estos recién nacidos se produce como resultado de la deficiencia de surfactante, desarrollo anatómico incompleto del pulmón, así como de la falta de madurez de otros sistemas. El uso de corticoides profilácticos en embarazos prematuros, acelera la maduración pulmonar y reduce la incidencia de SDR. Por lo tanto, el uso de corticoides prenatales se recomiendan actualmente entre las $24+0$ y $33+6$ semanas de gestación en mujeres con riesgo de parto prematuro dentro de los próximos siete días. La evidencia del uso de corticoides antenatales después de las 34 semanas sigue siendo debatible. Recientemente, el Colegio Americano de Obstetricia y Ginecología recomienda el uso de corticoides prenatales para mujeres con riesgo de parto prematuro de más de 34 semanas de gestación, pero no para las mujeres sometidas a cesárea electiva a término (2), mientras que el Real Colegio de Obstetras y Ginecólogos de Inglaterra recomiendan el uso de corticoides prenatales a todas la mujeres que serán sometidas a una cesárea electiva a edades gestacionales menores o iguales a 38+6 semanas (3). Esta revisión sistemática y meta-análisis, pretende evaluar la eficacia del uso de corticoides prenatales administrados en embarazos $\geq 34$ semanas para reducir la morbilidad respiratoria neonatal.

\section{B. El estudio (1)}

Diseño: Corresponde a una revisión sistemática y meta-análisis. La pregunta clínica es lógica y focalizada. Es una pregunta clara, bien elaborada y con sustento fisiopatológico. Esta revisión se realizó de acuerdo con un protocolo diseñado a priori basado en recomendaciones internacionales para realizar revisiones sistemáticas. Se realizaron búsquedas en Medline, Scopus, ClinicalTrials.gov, Embase, Sciencedirect, registro central de estudios controlados de la Cochrane Library y Scielo. No hubo restricción de idioma. La búsqueda fue realizada hasta febrero del 2016. Pacientes: Se incluyeron pacientes con embarazos únicos de 34 o más semanas de gestación que presentaron un parto prematuro tardío (entre las $34+0$ y 36+6) y las pacientes cursando embarazo $\geq 37$ semanas que fueron sometidas a una cesárea electiva sin trabajo de parto. Se analizaron seis estudios, con un total de 5.698 embarazos únicos. Tres de ellos incluyeron 3.200 pacientes entre las $34+0$ y $36+6$ semanas de gestación y con riesgo de parto prematuro inminente al momento del ingreso hospitalario. Los otros tres estudios incluyeron 2.498 mujeres sometidas a cesárea electiva a las $\geq 37$ semanas. Intervención y comparación: Se incluyeron todos los estudios controlados aleatorizados que comparan el uso de corticoides prenatales (grupo de intervención) versus ningún tratamiento esteroidal o placebo (grupo control), en mujeres con un embarazo único a las 34 semanas o más. También se incluyeron estudios en donde utilizaron corticoides prenatales, en pacientes que presentaron un parto prematuro tardío (entre las $34+0$ y las $36+6$ semanas) y embarazadas que fueron sometidas a una cesárea electiva a término ( $\geq 37$ semanas de gestación). Resultado primario: Incidencia de síndrome de distrés respiratorio severo. Resultados: El uso de un solo curso de corticoides (ya sean 12 mg de betametasona administrada intramuscularmente cada 24 horas por dos dosis o cuatro dosis de $6 \mathrm{mg}$ de dexametasona administrada por vía intramuscular cada 12 horas), reduce la incidencia de morbilidad respiratoria neonatal en las mujeres sometidas a cesárea electiva a las 37 semanas o más, así como en las mujeres en riesgo de parto prematuro inminente entre las entre las $34+0$ y las 36+6 semanas de gestación.

\section{B. Análisis crítico}

Validez interna: Fueron incluidos solo estudios randomizados aleatorizados sin restricción de idioma. Los criterios de inclusión son poco específicos y distintos entre los estudios. El trabajo solo hace referencia a que fueron incluidas pacientes entre las 34+0 y 36+6 semanas con riesgo de parto prematuro inminente al momento del ingreso y mujeres sometidas a cesárea electiva a las $\geq 37$ semanas. No evalúa las características demográficas de las pacientes incluidas en esta revisión. Los criterios de exclusión también están definidos genéricamente como estudios que utilizaron corticoides antes de las 34 semanas, aquellos que incluyen embarazos 
múltiples y estudios cuasi-aleatorizados (estudios con base pseudo-aleatoria como por ejemplo utilizando el número par o impar de hospitalización, fecha de nacimiento, etc.). La posibilidad de pérdida de estudios relevantes es baja ya que utiliza múltiples bases de datos diferentes para realizar la búsqueda (Medline, Scopus, ClinicalTrials.gov, Embase, Sciencedirect, registro central de estudios controlados de la Cochrane Library y Scielo), además de utilizar más de 20 términos relacionados para la búsqueda. Los seis estudios incluidos tienen un bajo riesgo de sesgo de asignación pero alto riesgo de sesgo tanto en participantes e investigadores ciegos respecto a la intervención, además de alto riesgo de sesgo en la obtención de la data para el análisis de los resultados. Se utilizó un análisis según intención de tratar. Los resultados de los seis estudios incluidos son consistentes ya que la variabilidad entre estudios en cuanto a la intervención y comparación es baja evaluado por medio del test de heterogeneidad $\mathrm{I}^{2}$. La evaluación de los estudios fueron reproducibles ya que fueron analizados por dos investigadores independientes. Las diferencias entre ellos fueron discutidas y resueltas en conjunto. Una de las limitaciones de este trabajo es que solo tres estudios fueron doble ciego. Otra limitación de este estudio es que no está descrito el intervalo óptimo entre la administración corticoidal y el parto para reducir el riesgo de morbilidad respiratoria neonatal. No existe análisis de sub-grupo según edad gestacional, peso neonatal, etc. Este trabajo no incorpora seguimiento a largo plazo de los neonatos y madres expuestas a terapia corticoidal.

Validez externa: Esta revisión sistemática y meta-análisis podría ser aplicable a nuestra población, ya que las intervenciones descritas para este sub-grupo de pacientes podrían ser aplicables en nuestros centros de salud. La conducta de administrar un curso corticoidal al grupo de embarazadas con riesgo de parto prematuro inminente entre las 34+0 y 36+6 semanas de gestación y las pacientes que serán sometidas a una cesárea electiva a las 37 semanas o más, es relativamente de bajo costo y accesible en todos los centros que atienden mujeres embarazadas en nuestro país. Esta intervención pareciera tener beneficios neonatales sin efectos adversos evidentes tanto para la madre o el embarazo.
Conclusiones: Revisión sistemática y metaanálisis de buena calidad metodológica ya que solo incluye estudios prospectivos aleatorizados controlados. Si bien la revisión sistemática no presenta problemas metodológicos, si observamos algunos problemas en los estudios incluidos. Existe riesgo de sesgo de intervención, información y de análisis debido a la falta de ciego de los investigadores y pacientes participantes en la mitad de los estudios incluidos. Los resultados de este trabajo hacen necesario discutir el uso de corticoides para reducir la morbilidad neonatal de los prematuros tardíos. Sin embargo, estos estudios solo consideran el potencial efecto benéfico, a corto plazo, de la terapia corticoidal, y no consideran los efectos a largo plazo que los corticoides pueden tener en el feto, como cambios metabólicos o endocrinos, que pueden facilitar la aparición de morbilidad crónica en el adulto $(4,5)$. Estimamos que es necesaria más información, relacionada con seguimiento a largo plazo de los niños prematuros tardíos nacidos luego de uso de corticoides, antes de considerar los resultados de esta investigación para un cambio de conducta en la clínica habitual.

\section{REFERENCIAS}

1. Saccone G, Berghella V. Antenatal corticosteroids for maturity of term or near term fetuses: systematic review and meta-analysis of randomized controlled trials. BMJ 2016;355:i5044.

2. Committee Opinion No. 677. ACOG committee opinion: antenatal corticosteroids therapy for fetal maturation. Obstet Gynecol 2016;128:e187-94.

3. Royal College of Obstetricians and Gynaecologists (RCOG). Antenatal corticosteroids to prevent respiratory distress syndrome. Clinical Green Top Guidelines. Royal College of Obstetricians and Gynaecologists, 2004.

4. Asztalos E. Antenatal corticosteroids: a risk factor for the development of chronic disease. J Nutr Metab 2012;2012:930591.

5. Asztalos E, Willan A, Murphy K, Matthews S, Ohlsson A, Saigal S, Armson A, Kelly E, Delisle MF, Gafni A, Lee S, Sananes R, Rovet J, Guselle P, Amankwah K; MACS-5 Collaborative Group. Association between gestational age at birth, antenatal corticosteroids, and outcomes at 5 years: multiple courses of antenatal corticosteroids for preterm birth study at 5 years of age (MACS-5). BMC Pregnancy Childbirth. 2014;14:272. 